\title{
Moluscos em área de horticultura no município de Porto Alegre, Rio Grande do Sul, Brasil ${ }^{1}$
}

\author{
Gisela Bruschi-Figueiró ${ }^{2,3}$ \\ Inga L. Veitenheimer-Mendes ${ }^{2}$
}

\begin{abstract}
Mollusca in horticultural production area in Porto Alegre county, Rio Grande do Sul, Brazil. Horticultural production areas are favorable to development and maintenance of land mollusks populations. To know the mollusk fauna diversity in that kind of production area a research project was conduced in a rural-urban district of Porto Alegre, Rio Grande do Sul, Brazil. The production property studied is located in the Southern zone of the city and has 17 ha of cultivated area. The sampling period was from August $/ 2000$ to August $/ 2001$, with monthly quantitative collects on the different culture production beds previously selected. Six taxa were registered: Deroceras leave (Müller, 1774) (1.389 specimens $=82,82 \%$ ), Bradybaena similaris (Férussac, 1821) (195 specimens $=11,7 \%$ ), Succinea meridionalis d'Orbigny, 1846 ( 82 specimens $=4,9 \%)$, Lamellaxis gracilis (Hutton, 1834) $(03$ specimens $=0,17 \%$ ), Bulimulus (Bulimulus) vesicalis angustus Weyrauch, 1966 (05 specimens $=0,3 \%$ ) and Megalobulimus abbreviatus (Bequaert, 1948) (02 specimens $=0,11 \%)$, only the last two being natives.

KEY WORDS. Deroceras laeve, Bradybaena similaris, landshells, horticulture, Porto Alegre
\end{abstract}

Culturas de hortaliças constituem-se em áreas propícias para o desenvolvimento e manutenção de moluscos terrestres - pela disponibilidade de alimento e umidade decorrente da irrigação. Literatura relativa ao registro de moluscos em horticultura é bastante restrita - FERNANDEZ DE VALERA (1982) registra danos causados por lesmas em horticultura na Venezuela.

No município de Porto Alegre, capital do Estado do Rio Grande do Sul, Brasil, está localizada a chamada cidade rururbana (PORTO AlEgRE 1999) que corresponde a aproximadamente $60 \%$ da área total do município. Essa área apresenta-se como um importante ponto de produção rural de hortigranjeiros, intercalando-se com núcleos de ocupação urbana. (HICKEL et al. 1998).

Desde 1997, o Laboratório de Malacologia da Universidade Federal do Rio Grande do Sul, vem desenvolvendo estudos relativos à fauna de moluscos em áreas urbanas de Porto Alegre, representadas pelos parques Farroupilha, Moinhos de Vento, Morro do Osso e pelo Jardim Botânico (VeITENHEIMER-Mendes \& AGUILAR-NUNES 2001).

1) Contribuição número 390 do Departamento de Zoologia, Universidade Federal do Rio Grande do Sul.

2) PPG-BAN, Departamento de Zoologia, Universidade Federal do Rio Grande do Sul. Avenida Bento Gonçalves 9500, prédio 43435, sala 201, 91501-970 Porto Alegre, Rio Grande do Sul, Brasil. E-mail: inga.mendes@ufrgs.br

3) Bolsista da CAPES. E-mail: gisela.bruschi@ufrgs.br 
Com o objetivo de conhecer a ocorrência e distribuição sazonal da fauna de moluscos terrestres em uma área de horticultura inserida na região urbana de Porto Alegre, Rio Grande do Sul, foram conduzidas coletas mensais quantitativas relacionando os resultados a 14 diferentes culturas da área pesquisada.

\section{MATERIAL E MÉTODOS}

O trabalho foi realizado em uma propriedade particular com cerca de 20 hectares, no bairro Lajeado, zona rururbana de Porto Alegre. Em 17 ha da propriedade são cultivados os seguintes culturas: espinafre da Nova Zelândia - Tetragonia tetragonioides Linnaeus (Aizoaceae), beterraba - Beta vulgaris Linnaeus (Chenopodiaceae), brócolos - Brassica oleracea Linnaeus var. italica Plenck (Cruciferae), couve-manteiga - B. oleracea Linnaeus var. acephala DC (Cruciferae), repolho B. oleracea Linnaeus var. capitata Linnaeus (Cruciferae), mostarda-de-folha Brassica juncea Coss (Cruciferae), rabanete - Raphanus sativus Linnaeus (Cruciferae), rúcula - Eruca sativa Linnaeus (Cruciferae), salsa - Petroselinum crispum Hoffm (Umbelliferae), alface - Lactuca sativa Linnaeus (Compositae), almeirão Chicorium intybus Linnaeus (Compositae), chicória - Chicorium endivia Linnaeus (Compositae), cebolinha-verde - Allium fistulosum Linnaeus (Liliaceae) e pimenta - Capsicum frutescens Linnaeus (Solanaceae).

Os moluscos foram amostrados a partir de coletas quantitativas mensais, de agosto de 2000 a agosto de 2001, durante o período da manhã. Para cada diferente cultura, presente no mês da amostragem, foi selecionado o canteiro de posição mais central da respectiva cultura; a coleta, restrita a quinze metros de cada canteiro, consistia no exame minucioso do solo e da parte aérea das plantas. De cada cultura analisada, foi colhida uma hortaliça para posterior exame em laboratório. Os moluscos coletados foram devidamente acondicionados e identificados por canteiro/cultura.

A estrutura da comunidade de moluscos foi determinada analisando-se a freqüência absoluta, a freqüência relativa e a constância. A constância (C) dos exemplares foi calculada, segundo DAJOZ (1983), onde $\mathrm{C}=\mathrm{p} \times 100 / \mathrm{P}$, em que $\mathrm{p}=$ número de coletas contendo a espécie estudada e $\mathrm{P}=$ número total de coletas efetuadas. A partir do valor de $\mathrm{C}$ distinguem-se as seguintes categorias: espécies constantes (presentes em mais de 50\% das coletas), espécies acessórias (presentes em 25 a $50 \%$ das coletas) e espécies acidentais (presentes em menos de $25 \%$ das coletas).

Em laboratório, os exemplares (ex.) foram identificados, quantificados, distendidos a frio conforme THOMÉ (1975), fixados e conservados em álcool a 70 GL e incluídos na coleção de moluscos do Departamento de Zoologia, Universidade Federal do Rio Grande do Sul (lotes 641, 642, 644-650, 692, 694, 696-699, 701, 707, 708, 710-715, 717, 749-752, 760-773).

\section{RESULTADOS E DISCUSSÃO}

Dos seis táxons de moluscos registrados para a área - Deroceras laeve (Müller, 1774), Limacidae com 1.389 ex. (82,82\%); Bradybaena similaris (Férussac, 1821), Xanthonychidae com 195 ex. (11,7\%); Succinea meridionalis d'Orbigny, 1846, Succineidae com 82 ex. (4,9\%); Lamellaxis gracilis (Hutton, 1834), Subulinidae com 03 ex. (0,17\%); Bulimulus (Bulimulus) vesicalis angustus 
Weyrauch, 1966, Bulimulidae com 05 ex. (0,3\%) e Megalobulimus abbreviatus (Bequaert, 1948), Megalobulimidae com 02 ex. $(0,11 \%)$ - apenas as duas últimas espécies são nativas.

ADOLFO-LÓPEZ \& PÉREZ (1996) afirmam que espécies exóticas são aquelas introduzidas por atividades humanas em áreas distantes de seu território natural. HARRY (apud ADOLFO-LÓPEZ \& PÉREZ 1996) propõe dois critérios para a introdução de espécies exóticas, isto é, a tendência de permanecerem em lugares afetados pela atividade humana e a falta de capacidade de povoarem locais distantes da área de introdução. Tais condições, provavelmente, se aplicam pelo menos a $D$. laeve e $B$. similaris, uma vez que, estas espécies têm sido registradas unicamente em áreas alteradas pelo homem, em especial, às de cultivos.

A distribuição sazonal de espécies de moluscos em relação ao número de exemplares mostrou os seguintes resultados (Fig. 1): D. laeve, B. similaris, $S$. meridionalis, $B$. vesicalis angustus e $M$. abbreviatus -142 ex. (8,47\% no inverno/2000); D. laeve, B. similaris, $S$. meridionalis e B. vesicalis angustus $-870 \mathrm{ex}$. (51,91\% na primavera/ 2000); D. laeve, B. similaris, S. meridionalis e L. gracilis 43 ex. (2,57\% no verão/2001); $D$. laeve, B. similaris e $S$. meridionalis - 89 ex. $(5,31 \%$ no outono/2001); D. laeve, B. similaris, S. meridionalis, B. vesicalis angustus, M. abbreviatus e L. gracilis - 532 ex. (31,74\% no inverno/2001).

Das 14 culturas analisadas, a pimenta (cultivada no verão e outono) foi à única na qual não foi registrada a presença de moluscos (Fig. 1).

No espinafre foram encontrados $D$. laeve e $B$. similaris como espécies constantes, além de $S$. meridionalis e $B$. vesicalis angustus (espécies acidentais) (Tab. I). Quanto à presença do Limacidae Agriolimax laevis (Müller, 1774) $(=D$. laeve), FERNANDEZ DE VALERA (1982), em pesquisa sobre lesmas de interesse agrícola na Venezuela, confirma o registro ocorrido na área avaliada, registrando esta espécie em apenas uma área com essa cultura.

Na cultura de brócolos foram registrados $D$. laeve (espécie constante); $S$. meridionalis e B. similaris (espécies acessórias); e, L. gracilis, B. vesicalis angustus e M. abbreviatus (espécies acidentais) (Tab. I). O registro de A. laevis por FERNANDEZ DE VALERA (1982), em uma única área de brócolos, corrobora mais uma vez com o registro na área de estudo.

A cultura de couve foi atacada por $D$. laeve (espécie constante); B. similaris e $S$. meridionalis (espécies acessórias); e, por B. vesicalis angustus (espécie acidental) (Tab. I). Tal fato, confirma o citado por CASTILLEJO et al. (1996) como problema à cultura de couve no que se refere à presença de limacídeos.

A cultura de alface foi atacada por $D$. laeve (espécie constante) e, por $B$. similaris e $S$. meridionalis (espécies acidentais) (Tab. I). FERNANDEZ DE VALERA (1982) registra A. laevis em três áreas de cultura de alface na Venezuela, enquanto que PAKARINEN et al. (1990) e CASTILLEJO et al. (1996) registram Limacidae do gênero Deroceras Rafinesque, 1820 - Deroceras agreste (Müller, 1774) na Europa Ocidental e Deroceras reticulatum (Müller, 1774) e Deroceras panormitanum (Lessona \& Pollonera, 1882) na Espanha, respectivamente, em culturas de alface.

Revta bras. Zool. 19 (Supl. 2): 31 - 37, 2002 


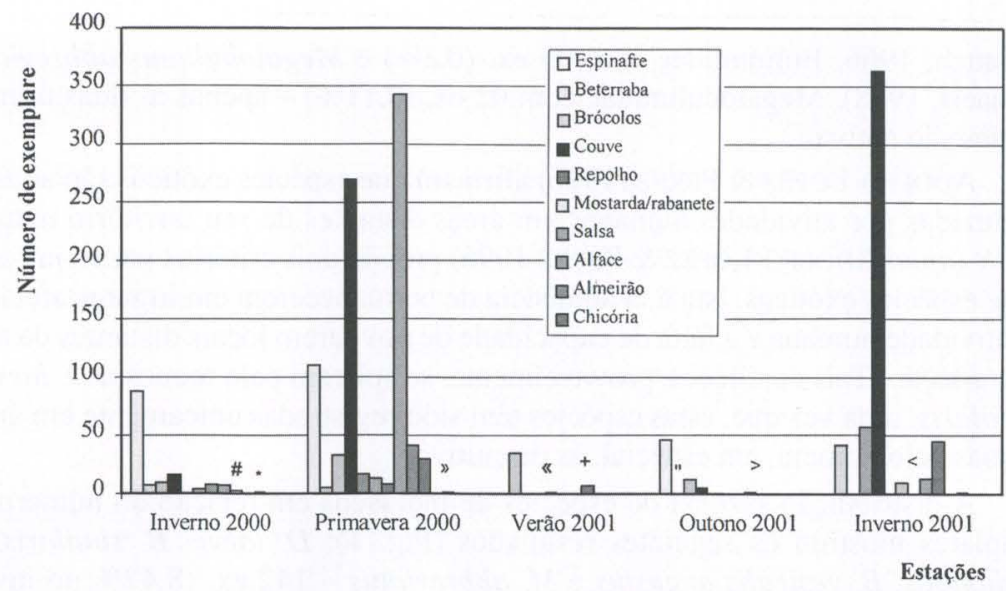

Fig. 1. Registro sazonal (inverno/2000 ao inverno/2001) de moluscos em uma área de horticultura no município de Porto Alegre, Rio Grande do Sul, Brasil. Legenda complementar: (\#) almeirão, (*) cebolinha, (") rúcula, (") mostarda/rabanete, (+) almeirão, (") beterraba, (>) alface, $(<)$ salsa.

Tabela I. Registro da constância - constantes, acessórias e acidentais - das espécies de moluscos coletadas nas diferentes hortaliças de uma área de horticultura no município de Porto Alegre, Rio Grande do Sul, Brasil, entre o inverno/2000 e o inverno/2001.

\begin{tabular}{|c|c|c|c|}
\hline \multirow{2}{*}{ Culturas } & \multicolumn{3}{|c|}{ Classificação } \\
\hline & Constantes (\%) & Acessórias (\%) & Acidentais (\%) \\
\hline Espinafre & $\begin{array}{l}\text { Deroceras laeve }(80) \\
\text { Bradybaena similaris }(80)\end{array}$ & & $\begin{array}{l}\text { Bulimulus vesicalis angustus (10) } \\
\text { Succinea meridionalis (20) }\end{array}$ \\
\hline Beterraba & & Deroceras laeve (43) & Succinea meridionalis (14) \\
\hline Brócolos & Deroceras laeve (77) & $\begin{array}{l}\text { Bradybaena similaris (46) } \\
\text { Succinea meridionalis ( } 38 \text { ) }\end{array}$ & $\begin{array}{l}\text { Bulimulus vesicalis angustus ( } 8 \text { ) } \\
\text { Megalobulimus abbreviatus ( } 8 \text { ) }\end{array}$ \\
\hline Couve & Deroceras laeve $(70)$ & $\begin{array}{l}\text { Bradybaena similaris }(38) \\
\text { Succinea meridionalis }(30)\end{array}$ & $\begin{array}{l}\text { Lamellaxis gracilis ( } 8 \text { ) } \\
\text { Bulimulus vesicalis angustus ( } 8 \text { ) }\end{array}$ \\
\hline Repolho & & Deroceras laeve (33) & \\
\hline Mostarda + rabanete & Deroceras laeve (55) & Bradybaena similaris (45) & Succinea meridionalis (18) \\
\hline Salsa & & Deroceras laeve (30) & Lamellaxis gracilis (10) \\
\hline Alface & Deroceras laeve (66) & & $\begin{array}{l}\text { Bradybaena similaris (16) } \\
\text { Succinea meridionalis (16) }\end{array}$ \\
\hline Almeirão & Deroceras laeve (80) & $\begin{array}{l}\text { Bradybaena similaris }(50) \\
\text { Succinea meridionalis }(50)\end{array}$ & Bulimulus vesicalis angustus (10) \\
\hline Chicória & & $\begin{array}{l}\text { Deroceras laeve (33) } \\
\text { Bradybaena similaris (33) }\end{array}$ & \\
\hline Cebolinha & & & $\begin{array}{l}\text { Deroceras laeve }(10) \\
\text { Bradybaena similaris }(10) \\
\text { Megalobulimus abbreviatus (10) }\end{array}$ \\
\hline Rúcula & & Deroceras laeve (50) & \\
\hline
\end{tabular}


Culturas de beterraba foram atacadas por $D$. laeve (espécie acessória) e $S$. meridionalis (espécie acidental) (Tab. I). FERNANDEZ DE VALERA (1982) registra A. laevis em quatro áreas de cultivo de beterraba na Venezuela; e, ESTER \& GEELEN (1996) registram ataque de lesmas às culturas de beterraba em experimentos na Holanda.

No repolho foram encontrados apenas exemplares de $D$. laeve (espécie acessória) (Tab. I), o que corrobora com os registros qualitativos desta espécie em dez áreas de cultivo de repolho na Venezuela por FERNANDEZ DE VALERA (1982).

Deroceras laeve, com o maior número de exemplares coletados, mostrou-se como espécie presente em todas as culturas atacadas por moluscos, mostrando-se constante em $50 \%$ das culturas, acessória em 41,67\% e acidental em 8,33\% (Tab. I). Espécies do gênero Deroceras são citadas na literatura atacando plantações: OlAZARRI (1979) e CAZZANIGA \& RONDA (1986) identificaram D. laeve para o Uruguai e Argentina, respectivamente; JAWORSKA (1993) registrou este gênero para a Polônia; Bolton et al. (1996), COOK et al. (1996, 1997) e FrANK \& FrIEDLI (1999) verificaram, para a Europa, D. reticulatum; HAMMOND (1996) e FRANK (1998) encontraram D. laeve e D. reticulatum na América do Norte.

Bradybaena similaris com o segundo maior número de moluscos coletados, mostrou-se presente em oito $(66,67 \%)$ das culturas atacadas por moluscos; apresentando-se constante em 8,33\% das culturas atacadas por moluscos, acessória em 41,67 \% e acidental em 16,67\% (Tab. I). É citada como praga agrícola por LOPES-PITONI et al. (1976), ARAÚJO (1989) e SANTOS (1994). No entanto, todos esses autores ressaltam a sua associação com helmintos, por ser vetora de parasitoses.

Não foi constatada uma relação de exclusividade entre molusco e hortaliça, uma vez que, exemplares de uma mesma espécie foram observados em diferentes hortaliças ao longo do ano (Tab. I). Entretanto, houve uma nítida dominância da lesma exótica $D$. laeve $(82,82 \%)$ em relação às demais espécies registradas para a área de estudo, confirmando observações feitas por diversos autores, para culturas em distintas regiões do mundo, sobre a ocorrência de $D$. laeve em diferentes hortaliças.

Danos causados por moluscos são difíceis de serem dimensionados pela falta de estudos básicos relativos à biologia dos mesmos (CASTILLEJO et al. 1996; FERNANDEZ DE VALERA 1982). No entanto, eventuais danos observados nas folhas de espinafre, couve e brócolos, pelo proprietário e durante o trabalho de campo, não podem ser atribuídos somente aos moluscos, uma vez que, também existem na área diferentes espécies de insetos.

Levantamentos de moluscos relativos a parques localizados na área urbana de Porto Alegre registram 17 espécies de moluscos terrestres - 13 nativas e quatro exóticas (informação verbal Carla Oliveira, Jakeline Aguilar-Nunes, Gisela Bruschi-Figueiró e Inga Veitenheimer-Mendes; VeITENHEIMER-MENDES \& AGUILARNunES 2001). Destas, três espécies exóticas (B. similaris, L. gracilis e D. laeve) e três espécies nativas ( $M$. abbreviatus, B. vesicalis angustus e $S$. meridionalis) também foram encontradas na área de horticultura, porém as exóticas em maior número do que o registrado para a área dos parques, sendo constatado o inverso para as três espécies nativas. 
AGRADECIMENTOS. Ao Sr. Paulo Neri Faria da Rosa, pelo apoio e permissão à realização do trabalho em sua propriedade de horticultura. Às colegas, do Laboratório de Malacologia do Departamento de Zoologia - UFRGS, Carla R. P. Oliveira, Cristiane M. S. Vianna, Jakeline Aguilar Nunes, Melissa Postal e Raquel I. Simões, pelo auxílio durante as coletas.

\section{REFERÊNCIAS BIBLIOGRÁFICAS}

Adolfo-LóPEZ, S.J. \& A.M. PÉREZ. 1996. Nuevos registros de caracoles terrestres "advenedizos" en Nicarágua: Leptinaria insignis, Bothriopupa conoidea y Caeciliodes iota (Mollusca: Gastropoda). Rev. Biol. Trop. 44 (1): 302-303.

ARaúso, J.L.B. 1989. Moluscos de importância econômica no Brasil. I. Xanthonychidae: Bradybaena similaris (Férussac, 1821), (Mollusca, Gastropoda, Pulmonata, Stylommatophora). Revta bras. Zool. 6 (4): 583-592.

Bolton, A.; L.D. InCOlL; S.G. Compton \& C. WRIGHT. 1996. The effect of management of rotacional set-aside on abundance and dispersion of slugs. Proceedings Symposium slug and snail pests in agriculture. Canterbury, Univ. Kent, p. 109-116.

CAstillejo, J.; L. SeiJas \& F. Villoch. 1996. Slug and snail pests in Spanish crops and their economical importance. Proceedings Symposium slug \& snail pests in agriculture. Canterbury, Univ. Kent, p. $327-332$.

Cazzaniga, N.J. \& C.D. Ronda. 1986. Las babosas Veronicellidae más australes de la Argentina (Gastropoda). Spheniscus 2: 19-22.

COOK, R.T.; S.E.R. BAILEY \& C.R. MCCROHAN. 1996. Slug preferences for winter wheat cultivars and common agricultural weeds. Jour. Appl. Ecol. 33: 866-872.

-1997. The potential for common weeds to reduce slug damage to winter wheat: laboratory and field studies. Jour. Appl. Ecol. 34: 79-87.

DAJOZ, R. 1983. Ecologia geral. Petrópolis, Ed. Vozes, 472p.

ESTER, A. \& P.M.T.M. GEELEN. 1996. Integrated control of slugs in a sugar beet crop growing in a rye cover crop. Proceedings Symposium slug \& snail pests in agriculture. Canterbury, Univ. Kent, p. $445-450$.

FERNANDEZ DE VALERA, J. 1982. Contribuicion al conocimiento de las babosas y sietecueros (Mollusca: Gastropoda) que causan daños a la agricultura en Venezuela. Rev. Fac. Agron., Maracay, 12 (3-4): 353-386.

FRANK, T. 1998. Slug damage and number of slugs (Gastropoda: Pulmonata) in winter wheat in fields with sown wildflower strips. Jour. Moll. Stud. 64: 319-328.

FRANK, T. \& J. FrIEDLI. 1999. Laboratory food choice trials to explore the potential of common weeds to reduce slug feeding on oilseed rape. Biol. Agric. Hortic., Bicester, 17: 19-29.

Hammond, R.B. 1996. Conservation tillage and slugs in the U. S. corn belt. Proceedings Symposium slug \& snail pests in agriculture. Canterbury, Univ. Kent, p. 31-38.

Hickel, H.T.; M.T.F. Albano; I.M.B. Pavlick \& D. BetTIOL. 1998. A organização urbana. p. 118. In: R. Menegat (Coord.). Atlas Ambiental de Porto Alegre - UFRGS-PMPA-INPE. Porto Alegre, Ed. da Universidade, 228p.

JAWORSKA, M. 1993. Laboratory infection of slugs (Gastropoda: Pulmonata) with entomopathogenic nematodes (Rhabditida: Nematoda). Jour. Inverteb. Pathol. 61: 223-224.

Lopes-Pitoni, V.L.; I.L. Veitenheimer \& M.C.D. Mansur. 1976. Moluscos do Rio Grande do Sul: coleta, preparação e conservação. Iheringia, Porto Alegre, 5: 25-68.

OlazARRI, J. 1979. Los moluscos plaga de los cultivos de "berro" en Salto, Uruguay. Com. Soc. malac. Urug. 5 (36): 63-69.

PAKARINEN, E.; P. NIEMELÄ \& J. TUOMI. 1990. Effect of fertilization, seaweed extracts and leaf-damage on palatability of lettuce to Deroceras-slugs. Acta Oecol. 11 (1): 113-19.

Porto Alegre (Prefeitura Municipal). 1999. Plano Diretor de Desenvolvimento Urbano Ambiental de Porto Alegre, Secretaria do Planejamento Municipal - Lei Complementar 434/99. Porto

Revta bras. Zool. 19 (Supl. 2): 31 - 37, 2002 
Alegre, SMAM, p. 34-35.

Santos, O. 1994. Presencia de Bradybaena similaris (Férussac, 1821) en el Uruguay. Com. Soc. malac. Urug. 7 (66-67): 376-378.

ThомÉ, J.W. 1975. Distensão de moluscos terrestres para fixação, com comentários sobre coleta e transporte. Arq. Mus. Nac., Rio de Janeiro, 55: 153-154.

Veitenheimer-Mendes, I.L. \& J. Aguilar-Nunes. 2001. Moluscos. p. 48-57. In: S.R. Mirapalhete

(Coord.). Flora e Fauna do Parque Natural Morro do Osso, Porto Alegre/RS. Porto Alegre, SMAM, 110p.

Recebido em 09.VII.2002; aceito em 04.XI.2002. 